\title{
Parche de sangre epidural torácico, de alto volumen, para el tratamiento del Síndrome de Hipotensión Intracraneana Espontánea secundario a fuga cervical de líquido cefalorraquídeo, ¿mejora el período asintomático?. Reporte de caso
}

PAOLA VIDAL MD. ${ }^{1 a b}$, ANDRÉS ROJAS MD, MG. ${ }^{1,2 a b}$, MÓNICA ROSAS MD. ${ }^{3 a}$, JULIO GARCÍA MD. ${ }^{4 a}$

\begin{abstract}
High blood volume thoracic epidural patch for the treatment of spontaneous intracranial hypotension syndrome due to cervical cerebrospinal fluid leak, It prolongs the asymptomatic period?. A case report

A 50-year-old woman, with a history of three-week orthostatic headache and nonspecific neurological symptoms, has a subdural hygroma at C5 level with a cerebrospinal fluid leakage compatible with Spontaneous Intracranial Hypotension Syndrome (SIHS). Initial conservative treatment has no response. A thoracic epidural blood patch (EBP) is performed, with a $20 \mathrm{ml}$ blood volume spread through an epidural needle, with a complete therapeutic response up-to 8 months. We believe that a higher blood volume patch through a thoracic approach gives the advantages of cervical and lumbar EBP, and, therefore, should be considered as a therapeutic alternative especially in technical anatomically difficult patients.
\end{abstract}

Key words:

Intracranial Hypotension, Cerebrospinal Fluid Leak, Blood Patch, Epidural, Headache.

\footnotetext{
Servicio de Anestesiología.

Magister en Epidemiologia Clínica.

Servicio de Neurología.

Servicio de Neurocirugía.

Hospital Puerto Montt, Chile.

Facultad de Medicina, Universidad San Sebastián.
}

Fecha de recepción: 10 de noviembre 2017

Fecha de aceptación: 8 de enero de 2018

ORCID

https://orcid.org/0000-0002-0634-1442

\section{Correspondencia:}




\section{RESUMEN}

Mujer de 50 años, con historia de 3 semanas de cefalea ortostática progresiva y síntomas neurológicos inespecíficos, confirmándose higroma subdural a nivel C5 con fuga de líquido cefalorraquídeo compatible con Síndrome de Hipotensión Intracraneal Espontánea (SHIE). Tratamiento médico inicial sin respuesta. Se realiza parche sanguíneo epidural (PSE) a nivel torácico con $20 \mathrm{ml}$ de sangre directo a través de trocar epidural, observándose respuesta terapéutica completa en seguimiento hasta 8 meses. Creemos que un PSE torácico ofrece las ventajas de uno cervical y lumbar y, por lo tanto, debe considerarse una alternativa terapéutica eficaz en este síndrome especialmente en pacientes anatómicamente complejos.

\section{Palabras clave:}

Hipotensión Intracranial, Pérdida de Líquido Cefalorraquídeo, Parche de Sangre Epidural, Cefalea.

\section{Introducción}

E Síndrome de Hipotensión Intracraneal Espontánea (SHIE) es una condición neurológica infrecuente, invalidante, relegada habitualmente a los diagnósticos secundarios de cefalea. Descrita por primera vez por Schaltenbrand, ocurre debido a una pérdida oculta de líquido cefalorraquídeo (LCR) en la columna vertebral, determinando así hipotensión y cefalea ortostática. Esta condición se relacionaría a pequeños quistes perineurales que podrían determinar desgarros de la duramadre, propiciando dicha pérdida[1].

Dada su fisiopatología, su sospecha diagnóstica es difícil, requiriendo habitualmente una evaluación e intervención multidisciplinaria, sumado a apoyo imagenológico[2].

Existen reportes de casos, la mayoría deficientes en cuanto a seguimiento, y con resultados terapéuticos dispares que van desde medidas conservadoras a la resolución quirúrgica[3]. Pareciera así que el uso de un parche sanguíneo epidural (PSE) pudiese corresponder al gold estándar de tratamiento para estos pacientes en términos de riesgo y beneficio.

Dado que la fuga de LCR es más frecuente a nivel cervical, los PSE lumbares tienen tasas de éxito más bajas, mientras que los cervicales, se asocian a una mayor efectividad al primer intento pero también a un mayor riesgo de neuropraxia (potencialmente devastadora)[4]. En este reporte de caso, presentamos los resultados y el seguimiento de un PSE torácico, de alto volumen sanguíneo, tratando de bloquear una fuga cervical en un paciente con una importante limitación anatómica.

\section{Consentimiento para publicación}

Este manuscrito se adhiere a la iniciativa CARE. Se contactó al paciente y se obtuvo el permiso por escrito para publicar este reporte de caso.

\section{Caso clínico}

Paciente de 50 años $(55 \mathrm{~kg}, 155 \mathrm{~cm}$ ), sexo femenino, sin antecedentes de importancia, hospitalizada por 3 semanas de cefalea progresiva fronto-occipital, espontánea, tipo ortostático, intensidad dolor 7/10 medida en escala visual analógica (EVA), asociada a náuseas, vómitos, fotofobia y tinnitus. Clínicamente sin signos de irritación meníngea, con signos vitales, examen físico y laboratorio normales. Se indica inicialmente tratamiento sintomático con metamizol, paracetamol, cafeína oral y corticosteroides endovenosos $(E V)$, todos los cuales determinaron escaso alivio del dolor.

Evaluada por equipo multidisciplinario, se realiza resonancia magnética (RM) cerebral que muestra higromas subdurales laminares bihemisféricos y paquimeningitis difusa (Figura 1A). Dado lo anterior, se realiza mielografía mediante tomografía axial computarizada (MieloTAC) que revela divertículo dural y fuga de LCR en C5 (Figura 1B).

Durante la tercera semana de hospitalización, y ante los hallazgos descritos, se solicita interconsulta a anestesiología para evaluar realización de PSE. En primera evaluación no queda claro componente ortostático, se constata además ansiedad importante y fotofobia, decidiéndose así ajustar analgesia, adicio- 


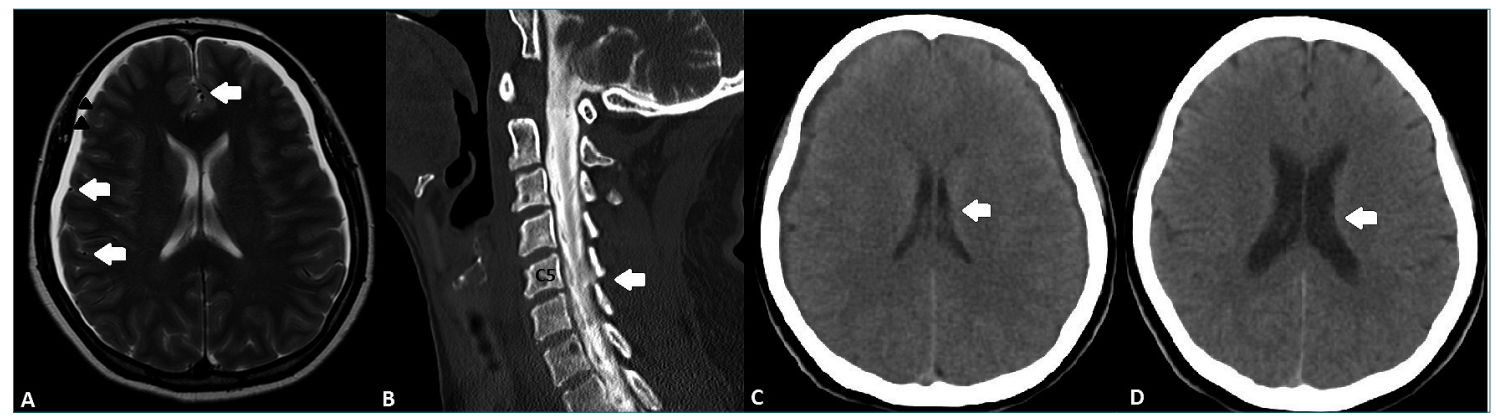

Figura 1. (A) RM cerebral T2, vista axial, mostrando higromas subdurales fronto-temporo-parietales bilaterales (flechas) y refuerzo paquimeningeo difuso (triángulos) compatibles con SHIE; (B) MieloTAC, vista sagital, mostrando fuga LCR a nivel C5; (C) TAC cerebro sin contraste, vista axial, 3 semanas previo a PSE, evidenciando disminución amplitud y volumen de ventrículos supratentoriales (flecha); (D) TAC cerebro sin contraste, vista axial, 2 meses post PSE, evidenciando aumento amplitud y volumen de ventrículos (flecha). RM: Resonancia magnética; SHIE: Síndrome hipotensión intracraneal espontánea; MieloTAC: mielograma mediante tomoagrafía axial computarizada; LCR: líquido cefalorraquídeo; TAC: Tomografía axial computarizada; PSE: Parche sanguíneo epidural.

nando antiepilépticos y benzodiacepinas y reevaluación en 48 horas, momento en el cual se constata cefalea EVA 3/10 y deambulación sin problemas, decidiéndose diferir PSE según evolución sintomatológica.

Cuatro días después, se solicita re-evaluación por anestesiología dado aumento cefalea, mayor componente postural, tinnitus y fotofobia. Se decide entonces realización de PSE, previa autorización paciente, a nivel torácico dada cifoescoliosis importante y ubicación del defecto dural (C5).

Se realiza procedimiento en pabellón con paciente en decúbito lateral izquierdo, monitoreo estándar, apoyo oxígeno y sedación con Midazolam 2 mg más Fentanilo 50 mcg EV. Bajo técnica estéril, y tras infiltración con lidocaína $2 \%$, se opta por punción a nivel T8-T9 mediante trocar epidural $18 \mathrm{G}$ (técnica de pérdida resistencia utilizando suero fisiológico). Se encuentra espacio epidural a $5 \mathrm{cms}$ y, manteniendo asepsia, enfermera asistente extrae $20 \mathrm{ml}$ de sangre autóloga desde vena braquial izquierda los cuales se administran inmediatamente a través de trocar.
Procedimiento total duró 15 minutos, sin incidentes asociados y transfiriendo a la paciente a unidad de recuperación post anestésica para monitorización atingente, manteniéndola en Trendelenburg por 30 minutos. Tras 2 horas, se reevalúa a paciente quien indica regresión de la cefalea así como de la sintomatología neurológica asociada descrita. No se describieron eventos adversos post procedimiento.

Completa 33 días hospitalizados, dándose de alta sin síntomas neurológicos tras 7 días post PSE. Evaluada clínica e imagenológicamente en diversas oportunidades (Figura 1c y 1d), manteniéndose asintomática hasta el día de hoy (8 meses seguimiento).

\section{Discusión}

La complejidad técnica que representa la pesquisa de esta patología, sumado a sus similitudes semiológicas con otras entidades neurológicas (meningitis, encefalitis, hemorragia subdural, cefalea post punción

\begin{tabular}{lll}
\multicolumn{2}{c}{ Tabla 1. Hallazgos en LCR, radiológicos y anatomopatológicos sugerentes de síndrome de hipotensión } \\
intracraneal espontánea
\end{tabular}

LCR: Líquido cefalorraquídeo; TAC: Tomografía axial computarizada; RM: Resonancia magnética. 


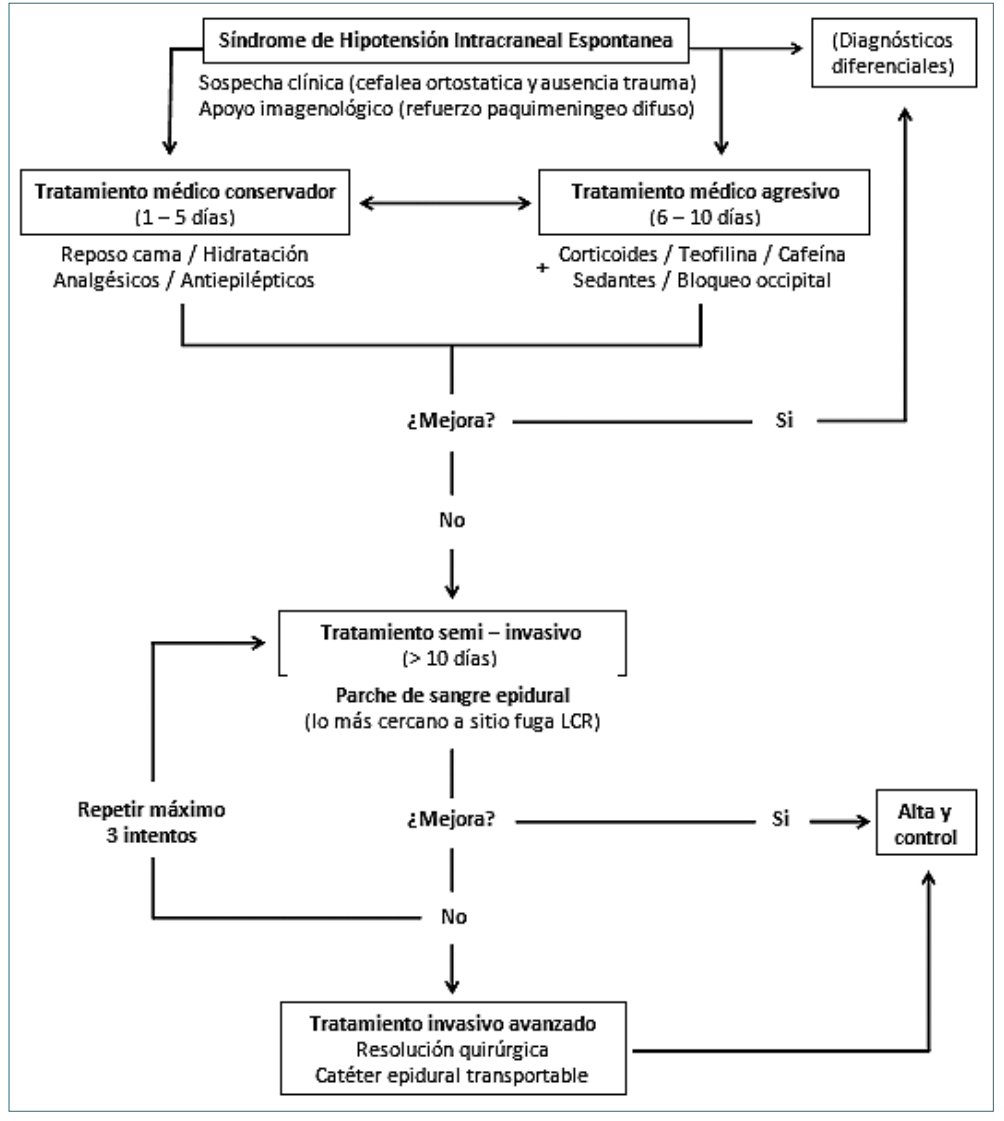

Figura 2. Flujograma propuesto para manejo de pacientes con sospecha de síndrome de hipertensión intracraneal espontánea. SHIE: Síndrome hipotensión intracraneal espontánea; LCR: Líquido Cefalorraquídeo. lumbar), explica la escasa información disponible al respecto[5]. Schievink, estimó una incidencia anual de 5 casos por cada 100.000 habitantes, afectando más a mujeres (relación 2:1) y con una edad de presentación promedio alrededor de los 40 años[6].

Los primeros informes de SHIE lo describieron como una "aliquorrea espontánea", apoyado por los hallazgos de Kunkle al eliminar el 10\% del volumen total de LCR en voluntarios sanos, determinando hipovolemia cerebral y reproduciendo las características de este síndrome (Tabla 1)[7].

A pesar de que su sospecha diagnóstica implica la ausencia de trauma dural conocido, su inicio puede ser insidioso, esto pues traumas menores podrían favorecer su desarrollo (caídas, tos, estornudo, actividad sexual) al producir la ruptura de quistes perineurales o espinales epidurales existentes, desgarrando la duramadre y determinando fuga de LCR. Esta hipovolemia-hipotensión secundarias originan, a través de un mecanismo de succión, un descenso del encéfalo, traccionando las estructuras de anclaje del cerebro, con vasodilatación meníngea compensatoria (refuer- zo paquimeningeo en la RM) y cefalea.

Clínicamente su presentación es muy heterogénea, desde cefalea ortostática hasta el coma, inclusive la muerte, debido a la respuesta vasodilatadora cerebral apareciendo derrames subdurales o higromas[8],[9].

El tratamiento del SHIE se basa, en primera instancia, en establecer medidas conservadoras que busquen aliviar los síntomas[10]. Otros tratamientos, como la infusión intratecal de solución salina, la colocación percutánea de sellador de fibrina o uso de catéter epidural transportable, siguen siendo experimentales[11]. La Figura 2 resume un enfoque terapéutico propuesto por nuestro grupo para abordar este síndrome. Los pacientes que no responden a estas intervenciones pueden beneficiarse de un PSE con sangre autóloga, lo que sugiere que la sangre inyectada sella el defecto de la duramadre mediante un efecto de taponamiento en el lugar de la fuga, aumentando la presión del espacio epidural por tiempo indeterminado[12].

Históricamente se ha utilizado el PSE a nivel lum- 


Tabla 2. Comparación de tres niveles de parche sanguíneo epidural en pacientes con síndrome hipotensión
intracraneal espontánea

PSE: Parche sanguíneo epidural; LCR: Líquido cefalorraquídeo.

bar, independientemente del sitio de fuga, con resultados dispares. Se usa con mayor frecuencia debido a la familiaridad derivada de la anestesia obstétrica y a un potencial menor riesgo de punción inadvertida de la duramadre. Sin embargo, y cuando se realiza en este nivel, a menudo se requiere más de un intento terapéutico (tasa de éxito del 70-75\% para el primer intento y hasta $90 \%$ para un tercero), pudiendo, paradójicamente, aumentar el riesgo de daño (hematoma espinal, neuropraxia medular)[13].

Dado lo anterior, múltiples autores sugieren que el sitio de fuga debe identificarse mediante imágenes y tratarse con un PSE focalizado (o lo más cercano posible), especialmente tras el fracaso de un primer intento lumbar[14]. La tasa de éxito del PSE aumenta también con el volumen de sangre utilizado, reportándose tasas de éxito del 80\% (10-15 ml) hasta 95\% $(20 \mathrm{ml})[12]$. A pesar de ello, se desconoce el volumen sanguíneo preciso a inyectar, y aún menos si existe una asociación entre volumen y período asintomático.

Con respecto a una eventual asociación entre la duración del PSE y el método de focalización, los resultados también son dispares. Wang, a través de un catéter epidural en C7-T1 para sellar un defecto a nivel C1-C2, informó 5 meses sin síntomas, sin embargo, utilizó múltiples parches de bajo volumen sanguíneo (3-5 ml)[9].

Girgis y cols., reportaron un caso parecido al nuestro, pero con una presentación más insidiosa. Utilizó $18 \mathrm{ml}$ de sangre directamente desde aguja epidural (a nivel T1-T2) para alcanzar un defecto C5, sin embargo, no informó seguimiento, desconociéndose resultados a largo plazo[15]. Zhang, en un paciente comatoso y con una fuga en T11-L2, utilizó un PSE en
T12-L1 con $18 \mathrm{ml}$ sangre a través de trocar epidural $22 \mathrm{G}$, informando respuesta terapéutica total pero sin seguimiento clínico[12].

A diferencia de estos estudios, nuestro caso no contó con soporte radiológico para precisar la ubicación de la fuga en el espacio intervertebral, lo cual sumado a la marcada desviación de la columna vertebral, limitó nuestros intentos de colocar un catéter epidural en el sitio de fuga. Dados nuestros resultados, creemos que el soporte radiológico es ideal pero no mandatorio para el procedimiento, sobre todo si utilizamos un volumen sanguíneo mayor, ampliando el área de cobertura y creando un PSE más denso (y eventualmente más duradero), reportándose hasta 36-50 meses libre de síntomas[10].

Si múltiples PSE fallan, debe considerarse entonces la reparación quirúrgica, reportándose hasta $100 \%$ de resolución de la cefalea por un período indefinido[15].

Teniendo en cuenta la escasa probabilidad de implementar estudios experimentales para evaluar el tratamiento óptimo en este grupo de pacientes, pareciese razonable entonces, y con la evidencia actual disponible, considerar al PSE como el estándar de oro para el tratamiento del SHIE en términos de riesgo y beneficio, dirigiendo la punción lo más cercanamente posible al sitio de fuga, maximizando así las probabilidades de éxito y evitando potenciales neuropraxias debido a la aguja misma o a un catéter epidural desplazado.

En ese sentido, y en especial para fugas cervicales, creemos que un PSE torácico combina las ventajas de otros abordajes (Tabla 2), recomendando así, y al menos en un primer intento, un volumen sanguíneo $>15$ $\mathrm{ml}$, pues pareciera prolonga el período asintomático. 


\section{Referencias}

1. Davidson B, Nassiri F, Mansouri A, et al. Spontaneous Intracranial Hypotension: A Review and Introduction of an Algorithm For Management. World Neurosurg 2017;101(3):343-49.

2. Silva $A E$, Pavan $P$, Oshima MM, Cardoso TM, Reis F. Intracranial hypotension secondary to spontaneous spinal cerebrospinal fluid leaks. Arq Neuropsiquiatr. 2017;75(3):201-02.

3. Idrissi AL, Lacour JC, Klein O, Schmitt E, Ducrocq X, Richard S. Spontaneous Intracranial Hypotension: Characteristics of the Serious Form in a Series of 24 Patients. World Neurosurg. 2015;84(6):1613-20.

4. Ansel S, Rae A, Tyagi A. Efficacy of epidural blood patches for spontaneous lowpressure headaches: a case series. J R Coll Physicians Edinb. 2016;46(4):234-37.

5. Kingston W, Hoxworth J, HalkerSingh R. Spontaneous intracranial hypotension diagnosed as Chiari I malformation. Neurolo- gy. 2017;88(13):1294.

6. Schievink WI. Spontaneous spinal cerebrospinal fluid leaks and intracranial hypotension. JAMA 2006;295(19):2286-96.

7. Kunkle EC, Ray BS, Wolf HG. Experimental studies on headaches: analysis of the headache associated with changes in intracranial pressure. Arch Neurol Psychiatry 1943; 49:323-58.

8. Collange $\mathrm{O}$, Wolff $\vee$, Cebula $\mathrm{H}$, y cols. Spontaneous Intracranial Hypotension: An Etiology for Consciousness Disorder and Coma. AA Case Rep. 2016;7(10):207-11.

9. Wang E, Wang D. Successful treatment of spontaneous intracranial hypotension due to prominent cervical cerebrospinal fluid leak with cervical epidural blood patch. Pain Med. 2015;16(5):1013-18.

10. Davidson B, Nassiri F, Mansouri A, y cols. Spontaneous Intracranial Hypotension: A Review and Introduction of an Algorithm For Management. World Neurosurg 2017; 101(3):343-49.

11. Schievink WI, Rosner HL,
Louy C. A wearable epidural catheter infusion system for patients with intractable spontaneous intracranial hypotension. Reg Anesth Pain Med 2015;40(1):49-51.

12. Zhang J, Jin D, Pan KH. Epidural blood patch for spontaneous intracranial hypotension with chronic subdural haematoma: A case report and literature review. J Int Med Res. 2016;44(4):97681.

13. Graff-Radford SB, Schievink WI. High-pressure headaches, low-pressure syndromes, and CSF leaks: diagnosis and management. Headache 2014;54(2):394-01.

14. Nipatcharoen P, Tan SG. High thoracic/cervical epidural blood patch for spontaneous cerebrospinal fluid leak: a new challenge for anesthesiologists. Anesth Analg 2011;113(6):1476-79.

15. Girgis F, Shing M, Duplessis S. Thoracic epidural blood patch for spontaneous intracranial hypotension: case report and review of the literature. Turk Neurosurg. 2015;25(2):320-25 